\title{
Teaching NeuroImages: Extrapontine osmotic demyelination in hypernatremia
}

Pablo Paz, MD, Jie Pan, MD, PhD, and Somedeb Ball, MD

Neurology ${ }^{\circledR}$ 2020;94:e1780-1781. doi:10.1212/WNL.0000000000009299

Figure Diffusion-weighted imaging (DWI), apparent diffusion coefficient (ADC), and fluidattenuated inversion recovery (FLAIR)

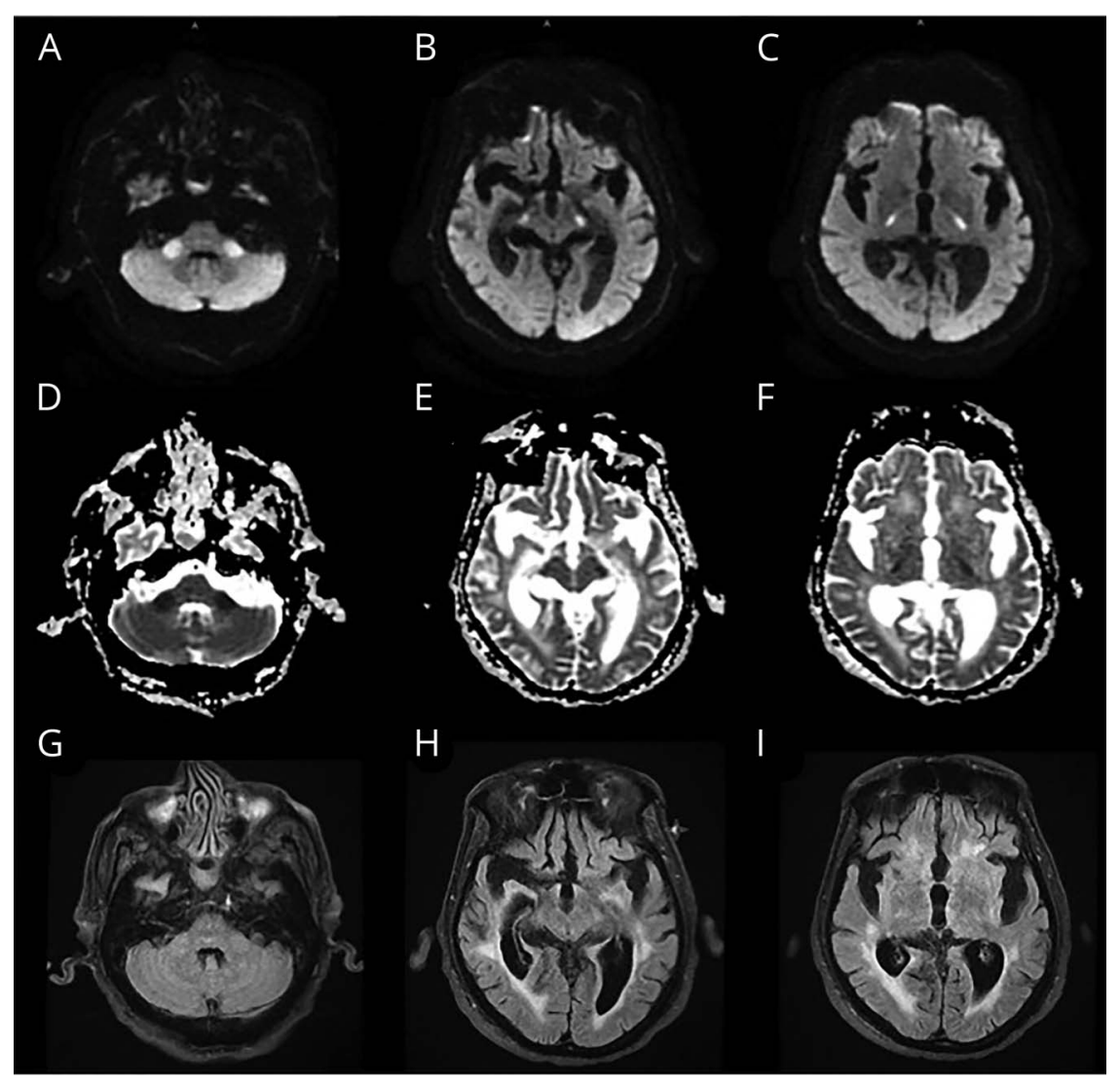

Axial diffusion-weighted MRI brain shows bilateral symmetrical hyperintensities in (A) middle cerebral peduncles and $(B, C)$ along the cortical spinal tracts. (D-F) ADC sequence shows areas of hypodensity correlating with hyperintensity in DWI sequence. (G-I) Subtle changes noticed in FLAIR sequence due to the early stage of demyelination process.

An 82-year-old man presented with altered mental status, withdrawal to pain, osmolality 405 $\mathrm{mOsm} / \mathrm{kg}$, and sodium $>180 \mathrm{mEq} / \mathrm{L}$, corrected at $8 \mathrm{mEq} / \mathrm{L} / \mathrm{d}$. Brain MRI on day 4 showed restricted diffusion within bilateral middle cerebellar peduncles and along the cortical spinal tracts (figure). Extrapontine osmotic demyelination was diagnosed. Classically described after rapid correction of hyponatremia, few cases have been reported in patients with severe hypernatremia. Extrapontine osmotic demyelination occurs after rapid increase in extracellular sodium causing cellular dehydration and cell death of astrocytes or oligodendrocytes provoking non-inflammatory demyelinating lesions in pontine or extrapontine regions. Isolated extrapontine lesions are seen in two-fifths of patients. ${ }^{1,2}$
Correspondence

Dr. Pan

jie.pan@ttuhsc.edu
MORE ONLINE

\section{$\rightarrow$ Teaching slides}

links.lww.com/WNL/ B74

From the Departments of Internal Medicine (P.P., S.B.) and Neurology (.P.), Texas Tech University Health Science Center, Lubbock.

Go to Neurology.org/N for full disclosures. Funding information and disclosures deemed relevant by the authors, if any, are provided at the end of the article. 


\section{Study funding}

No targeted funding reported.

\section{Disclosure}

P. Paz, J. Pan, and S. Ball report no relevant disclosures. Go to Neurology.org/ $\mathrm{N}$ for full disclosures.

\section{Appendix Authors}

\begin{tabular}{lll}
\hline Name & Location & Contribution \\
\hline $\begin{array}{l}\text { Pablo Paz, } \\
\text { MD }\end{array}$ & $\begin{array}{l}\text { Texas Tech University } \\
\text { Health Science Center, } \\
\text { Lubbock }\end{array}$ & $\begin{array}{l}\text { Literature review, } \\
\text { paper writing }\end{array}$ \\
& \\
\hline
\end{tabular}

Appendix (continued)

\begin{tabular}{lll}
\hline Name & Location & Contribution \\
\hline $\begin{array}{ll}\text { Jie Pan, } \\
\text { MD, PhD }\end{array}$ & $\begin{array}{l}\text { Texas Tech University Health } \\
\text { Science Center, Lubbock }\end{array}$ & $\begin{array}{l}\text { Literature review, } \\
\text { image processing }\end{array}$ \\
\hline $\begin{array}{l}\text { Somedeb } \\
\text { Ball, MD }\end{array}$ & $\begin{array}{l}\text { Texas Tech University Health } \\
\text { Science Center, Lubbock }\end{array}$ & Literature review \\
\hline
\end{tabular}

\section{References}

1. Jee Han M, Hyoung Kim D, Hwa Kim Y, Mo Yang I, Hyung Park J, Ki Hong M. A case of osmotic demyelination presenting with severe hypernatremia. Electrolyte Blood Press 2015;13:30-36.

2. Barhaghi K, Molchanova-Cook O, Rosenburg M, et al. Osmotic demyelination syndrome revisited: review with neuroimaging. J LA State Med Soc 2017;169:89-93. 


\section{Neurology}

\section{Teaching NeuroImages: Extrapontine osmotic demyelination in hypernatremia}

Pablo Paz, Jie Pan and Somedeb Ball

Neurology 2020;94;e1780-e1781 Published Online before print March 27, 2020

DOI 10.1212/WNL.0000000000009299

This information is current as of March 27, 2020

\section{Updated Information \& Services}

References

Subspecialty Collections

Permissions \& Licensing

Reprints including high resolution figures, can be found at: http://n.neurology.org/content/94/16/e1780.full

This article cites 2 articles, 0 of which you can access for free at: http://n.neurology.org/content/94/16/e1780.full\#ref-list-1

This article, along with others on similar topics, appears in the following collection(s):

MRI

http://n.neurology.org/cgi/collection/mri

Information about reproducing this article in parts (figures,tables) or in its entirety can be found online at:

http://www.neurology.org/about/about_the_journal\#permissions

Information about ordering reprints can be found online: http://n.neurology.org/subscribers/advertise

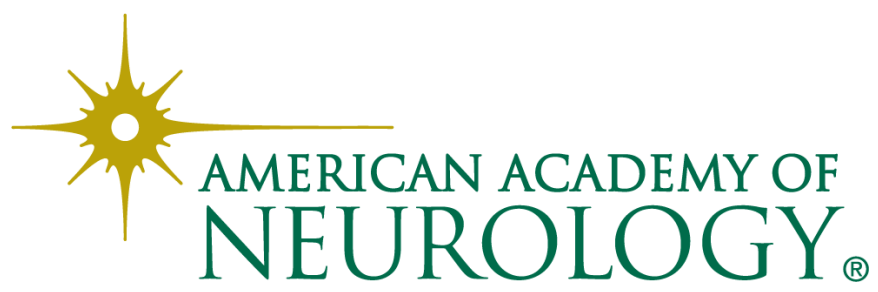

